\title{
Quantum tunneling, adiabatic invariance and black hole spectroscopy
}

\author{
Guo-Ping Li ${ }^{1, \mathrm{a}}$, Jin Pu ${ }^{1,2, \mathrm{~b}}$, Qing-Quan Jiang ${ }^{2, \mathrm{c}}$, Xiao-Tao $\mathbf{Z} \mathbf{u}^{1, \mathrm{~d}}$ \\ ${ }^{1}$ School of Physical Electronics, University of Electronic Science and Technology of China, Chengdu 610054, China \\ ${ }^{2}$ College of Physics and Space Science, China West Normal University, Nanchong 637009, China
}

Received: 30 March 2017 / Accepted: 8 May 2017 / Published online: 17 May 2017

(C) The Author(s) 2017. This article is an open access publication

\begin{abstract}
In the tunneling framework, one of us, Jiang, together with Han has studied the black hole spectroscopy via adiabatic invariance, where the adiabatic invariant quantity has been intriguingly obtained by investigating the oscillating velocity of the black hole horizon. In this paper, we attempt to improve Jiang-Han's proposal in two ways. Firstly, we once again examine the fact that, in different types (Schwarzschild and Painlevé) of coordinates as well as in different gravity frames, the adiabatic invariant $I_{\text {adia }}=\oint p_{i} d q_{i}$ introduced by Jiang and Han is canonically invariant. Secondly, we attempt to confirm Jiang-Han's proposal reasonably in more general gravity frames (including Einstein's gravity, EGB gravity and HL gravity). Concurrently, for improving this proposal, we interestingly find in more general gravity theories that the entropy of the black hole is an adiabatic invariant action variable, but the horizon area is only an adiabatic invariant. In this sense, we emphasize the concept that the quantum of the black hole entropy is more natural than that of the horizon area.
\end{abstract}

\section{Introduction}

The black hole as a special object with the strong gravitational field, has always been regarded as a test bed for any proposed scheme for a quantum-gravity theory. It therefore shows in principle that the exploration of the black hole entropy/area quantum has great significance, since it may provide a window on finding an effective way to quantize a gravitational field. However, there is as yet no complete quantum theory of gravity. Hence, reenforcing our understanding of these issues at a semiclassical level may be an appropriate juncture. In the

\footnotetext{
a e-mail: gpliphys@yeah.net

be-mail: pujin13222@163.com

ce-mail: qqjiangphys@yeah.net

de-mail: xtzu@uestc.edu.cn
}

context of a semiclassical notion, Bekenstein has reported that black hole entropy is always quantized in units of $\ell_{p}^{2}$ [3], ${ }^{1}$ and the quantum of the horizon area usually appears in the form $\Delta A=8 \pi \ell_{p}^{2}$ (where $\ell_{p}=\sqrt{\hbar G / c^{3}}$ is the Planck length) [7]. This idea mainly comes from the remarkable fact that the black hole area in the nonextremal case is an adiabatic invariant [8-11]. Later on, various types of other semiclassical approaches have appeared to further study the quantization of the black hole entropy [12-52]. The most popular one among them was proposed by Kunstatter in 2002. It is pointed out that, for a given system (black hole) equipped with the energy $E$ and the vibrational frequency $\omega(r)$, the action $I=\int \mathrm{d} E / \omega(E)$ is an adiabatic invariant [12]. Then, by using the Bohr correspondence principle for the quasinormal frequency, the entropy spectrum of a d-dimensional spherically symmetric black hole is equally spaced with its quantum to be equal to the one given by Hod [13,14], as well as by Bekenstein and Mukhanov [15]. ${ }^{2}$ However, in the Kunstatter's treatment, if one employs the oscillating frequencies of the black hole only coming from the real part of the highly damped quasinormal frequencies, there is an illdefined (adiabatic invariant) integral with inclusion of a large logarithmic term when it is used with the case of the rotating black hole [24]. In 2008, Maggiore has suggested that the black hole can be treated as a damped harmonic oscillator, and correspondingly, it is relevant that the frequency should contain contributions from both real and imaginary parts of the complex quasinormal mode frequencies, rather than only from the real part [23]. Following this new explanation and the black hole property of adiabaticity, it has been shown that the equally spaced area spectrum of a slowly rotating black hole can be expressed as the form $\Delta A=8 \pi \ell_{p}^{2}$ [25], which is exactly equal to the original result of Beken-

\footnotetext{
1 This was also confirmed by several different approaches [4-6].

${ }^{2}$ Here, a lot of work in this direction can be found in [15-22] and the references therein.
} 
stein [7]. Later, much further work has shown that the black hole spectroscopy in more general gravity frames can also be properly reproduced by combining the new explanation for quasinormal mode frequency and the black hole property of adiabaticity [24-35,49-52].

Recently, the interesting notion has been found that, without the aid of quasinormal modes, one can quantize the entropy and horizon area of the black hole in the tunneling mechanism [2]. ${ }^{3}$ Here, the action $I=\int p_{i} \mathrm{~d} q_{i}$ is an adiabatic invariant, which can be intriguingly obtained by analyzing the tunneling behavior of the emitted particle. However, one of us, Jiang, has found that this adiabatic invariant proposed in [2] is not canonically invariant [1]. Introducing a covariant form for the adiabatic invariant, Jiang and Han have combined the oscillating velocity of the black hole horizon to revisit the black hole spectroscopy of the Schwarzschild black hole in the tunneling picture [1]. It has been shown that the area spectrum of the Schwarzschild black hole is described with an equally spacing $\Delta A=8 \pi \ell_{p}^{2}$, which is in agreement with Bekenstein's original proposal [7] and is fully in consistence with the previous findings by combining the black hole property of adiabaticity and the black hole quasinormal frequencies [24-35,49-52].

It is necessary to further improve Jiang-Han's proposal to discuss the black hole spectroscopy in the tunneling framework. In this paper, we attempt to perfect this proposal in the two ways. Firstly, we once again examine, in different types (Schwarzschild and Painlevé) of coordinates as well as in different gravity frames, the fact that the adiabatic invariant $I_{\text {adia }}=\oint p_{i} \mathrm{~d} q_{i}$ introduced by Jiang and Han is canonically invariant, and it is more universal than the one $I_{\text {adia }}=\int p_{i} \mathrm{~d} q_{i}$ given by Majhi and Vagenas. Secondly, we generalize Jiang-Han's work from the Schwarzschild black hole to the case of more general gravity frames (including Einstein's gravity, EGB gravity and HL gravity). Our results are fully in consistence with the previous findings by combining the black hole property of adiabaticity and the black hole quasinormal frequencies [49-52]. Jiang-Han's proposal has been reasonably confirmed in more general gravity frames. Concurrent with improving this proposal, we interestingly find in more general gravity frames that the entropy of the black hole is an adiabatic invariant action variable, but the horizon area is only an adiabatic invariant. In this sense, we emphasize the concept that the quantum of the black hole entropy is more natural than that of the black hole area.

The remainder of this paper is outlined as follows. In Sect. 2, by investigating the adiabatic invariant quantity in different types (Schwarzschild and Painlevé) of coordinates as well as in different gravity frames, we attempt to examine the fact that the adiabatic invariant $I_{\text {adia }}=\oint p_{i} \mathrm{~d} q_{i}$ intro-

\footnotetext{
3 Some related work with the tunneling mechanism can be found in [53-120] and the references therein.
}

duced by Jiang and Han is canonically invariant. Using this covariant adiabatic invariant, Sect. 3 is devoted to the study of the entropy/area spectrum in different gravity frames (including Einstein's gravity, EGB Gravity and HL gravity). Section 4 proves that the entropy of the black hole is an adiabatic invariant action variable, but the horizon area is only an adiabatic invariant. Section 5 ends with a discussion and conclusion.

\section{Adiabatic invariant in different coordinates}

According to [1], it has been shown that the adiabatic invariant $I_{\text {adia }}=\oint p_{i} \mathrm{~d} q_{i}$ is canonically invariant. In this section, we aim to examine this fact by discussing the adiabatic invariant quantity in different types (Schwarzschild and Pain-levé) of coordinates, as well as in different gravity frames.

\subsection{Adiabatic invariant in the schwarzschild coordinate}

For a black hole in different gravity, without loss of generality, we only focus on the case of the spherically symmetric static solution. At present, it is generally believed that the near-horizon theory of the black hole can be reduced to the effective $(1+1)$-dimensional theory with gauge potential with respect to the electric gauge symmetry and/or the rotating symmetry. Therefore, it is reasonable to focus our attention on the case of the spherically symmetric static solution without loss of generality. The generally spherically symmetric black hole is always described by

$\mathrm{d} s^{2}=-F(r) \mathrm{d} t^{2}+\frac{\mathrm{d} r^{2}}{G(r)}+r^{2} \mathrm{~d} \Omega_{n-2}^{2}$.

For a black hole in different gravity, the metric (1) has the same form, only $F(r), G(r)$ taking different forms. Obviously, the surface gravity of (1) can be expressed as $\kappa=$ $\left.\frac{1}{2} \sqrt{\partial_{r} F(r) \partial_{r} G(r)}\right|_{r=r_{h}}$ [2], where $r_{h}$ is the event horizon of black hole.

Case I In Einstein gravity, a spherically symmetric black hole solution with a global monopole is characterized by the metric $[121,122]$

$F(r)=G(r)=\left(1-8 \pi \eta_{0}^{2}-\frac{2 M}{r}\right)$

where $n=4, \mathrm{~d} \Omega_{2}$ represents the two-dimensional spherical hypersurface, $M$ is the mass of the black hole, and $\eta_{0}$ is the symmetry breaking scale when the monopole is formed [121]. The event horizon is determined by the equation $F(r)=0$, which results in $r_{h}=2 M /\left(1-8 \pi \eta_{0}^{2}\right)$. It is well known that the area $A$ in Einstein gravity occurs in 
$S=\frac{A}{4 \ell_{p}^{2}}$.

Case II The Einstein-Gauss-Bonnet (EGB) gravity emerges as a low-energy limit of the supersymmetric string theory, which can be regarded as an effective model of gravity in higher dimensions. Here, an exact and spherically metric solution has been given by Boulware and Deser [123],

$F(r)=G(r)=\left[1+\frac{r^{2}}{2 \alpha}\left(1-\sqrt{1+\frac{16 \alpha M}{r^{4}}}\right)\right]$

where $n=5, \mathrm{~d} \Omega_{3}$ represents the three-dimensional spherical hypersurface, and $\alpha$ is the Gauss-Bonnet constant. The radius of the event horizon $r_{h}$ is given by $r_{h}=\sqrt{4 M-\alpha}$. For the metric (4), the relationship between the black hole entropy and the horizon area is [50-52]

$S=\frac{A}{4 \ell_{p}^{2}}\left[1+6 \alpha\left(\frac{A}{\Sigma_{3}}\right)^{-2 / 3}\right]$

where the $\Sigma_{3}$ is the volume of the unit 3-sphere. Unlike the case of Einstein gravity, it should be pointed out that the black hole entropy in the context of EGB gravity is no longer proportional to the horizon area. In general, their relationship is of the form of a function $A=f(S)$ which is not linear.

Case III Inspired by the Lifshitz model in condensed matter physics, a power counting renormalizable theory for the $(3+1)$-dimensional quantum gravity was proposed by Hořava $[124,125]$. This theory is popularly known as "Hořava-Lifshitz(HL) gravity". Employing the deformed action in the limit $\lambda=1, \Lambda_{W} \rightarrow 0$ [126], a static and asymptotically flat black hole solution is given by [127]

$F(r)=G(r)=\left(1+\omega r^{2}\left(1-\sqrt{1+\frac{4 M}{\omega r^{3}}}\right)\right)$.

Here, $n=4$, this solution is the so called the "KehagiasSfetsos" black hole, and the event horizon is $r_{h}=M$ $\left(1+\sqrt{1-\frac{1}{2 \omega M^{2}}}\right)$, and the parameter $\omega$ represents the feature of the Hořava-Lifshitz spacetime. For the metric (6), as discussed by Myung [128,129], a compact expression of the entropy in the context of HL gravity is presented thus:

$S=\frac{A}{4 \ell_{p}^{2}}\left(1+\frac{4 \pi}{A \omega} \ln \left[\frac{A}{4}\right]\right)$.

Similarly to the case of EGB gravity, the relationship between black hole entropy and area in the context of HL gravity is also not linear.

Now, let us focus on studying the adiabatic invariant quantity in the tunneling framework. To correctly address the black hole spectroscopy via adiabatic invariance, Jiang argued that the adiabatic invariant quantity should be of the covariant form [1]

$I_{\text {adia }}=\oint p_{i} \mathrm{~d} q_{i}=\int_{q_{i}^{\text {in }}}^{q_{i}^{\text {out }}} p_{i}^{\text {out }} \mathrm{d} q_{i}+\int_{q_{i}^{\text {out }}}^{q_{i}^{\text {in }}} p_{i}^{\text {in }} \mathrm{d} q_{i}$,

where the closed contour integral can be understood as a closed path that from one point $\left(q_{i}=q_{i}^{\text {out }}\right.$, just outside the horizon) to another point $\left(q_{i}=q_{i}^{\text {in }}\right.$, just inside the horizon) near the horizon. $q_{i}^{\text {out }}\left(q_{i}^{\text {in }}\right)$ and $p_{i}^{\text {out }}\left(p_{i}^{\text {in }}\right)$ stand for the coordinate and the canonical momentum, respectively. For a spherically symmetric black hole horizon, $q_{i}$ corresponds to the Euclidean time $\tau$ and the black hole horizon $r_{h}$. Using the Hamilton canonical equation $\dot{q}_{i}=\frac{\mathrm{d} H}{\mathrm{~d} p_{i}}$, one can see that $p_{i}^{\text {out }}$ and $p_{i}^{\text {in }}$ have the same absolute value, but opposite signs. In this sense, we have

$$
\begin{aligned}
\oint p_{i} \mathrm{~d} q_{i} & =2 \int_{q_{i}^{\text {out }}}^{q_{i}^{\text {in }}} p_{i}^{\text {in }} \mathrm{d} q_{i}=2 \iint_{0}^{H} \frac{\mathrm{d} H^{\prime}}{\dot{q}_{i}} \mathrm{~d} q_{i} \\
& =2\left(\int_{\tau_{\text {out }}}^{\tau_{\text {in }}} \int_{0}^{H} \mathrm{~d} H^{\prime} \mathrm{d} \tau+\int_{r_{\text {out }}}^{r_{\text {in }}} \int_{0}^{H} \frac{\mathrm{d} H^{\prime}}{\dot{r}_{h}} \mathrm{~d} r_{h}\right) \\
& =4 \int_{r_{\text {out }}}^{r_{\text {in }}} \int_{0}^{H} \frac{\mathrm{d} H^{\prime}}{\dot{r}_{h}} \mathrm{~d} r_{h} .
\end{aligned}
$$

Here, $\dot{r}_{h} \equiv \frac{\mathrm{d} r_{h}}{\mathrm{~d} \tau}$, and $H$ represents the Hamiltonian of a black hole. Obviously, to perform the integral (9), we should find the oscillating velocity of the black hole horizon in the first place. In the tunneling picture, when a particle tunnels out or in the black hole horizon, the black hole horizon will shrink or expand at the same time due to the loss or gain of the black hole mass [130]. It is evident that the particle's tunneling velocity $\dot{r}$ in this sense is equal and opposite to the oscillating velocity of the black hole horizon $\dot{r_{h}}$ [131], which is

$\dot{r_{h}}=-\dot{r}$.

Specifically, for a spherically symmetric spacetime, after Euclideanizing the metric (1) by the transformation $t=-i \tau$, we have $\mathrm{d} s^{2}=F(r) \mathrm{d} \tau^{2}+\frac{\mathrm{d} r^{2}}{G(r)}+r^{2} \mathrm{~d} \Omega_{n-2}^{2}$. In this case, when a photon travels across the black hole horizon, the radial geodesics turn out to be

$\dot{r} \equiv \frac{\mathrm{d} r}{\mathrm{~d} \tau}= \pm i F(r)$

with the upper(lower) sign of $\dot{r}$ corresponding to the outgoing/ingoing paths. Therefore, the oscillating velocity of the black hole horizon is

$\dot{r}_{h}=-\dot{r}=\frac{\mathrm{d} r_{h}}{\mathrm{~d} \tau}=\mp i F(r)$. 
Substituting (12) into the integral (9), then the adiabatic invariant quantity (8) can be finally written as

$I_{\text {adia }}^{S}=4 \int_{r_{\text {out }}}^{r_{\text {in }}} \int_{0}^{H} \frac{\mathrm{d} H^{\prime}}{\dot{r}_{h}} \mathrm{~d} r_{h}=-4 i \int_{r_{\text {out }}}^{r_{\text {in }}} \int_{0}^{H} \frac{\mathrm{d} H^{\prime}}{F(r)} \mathrm{d} r$,

where we have implemented $\dot{r}_{h}=-\dot{r}$ and $\mathrm{d} \dot{r}_{h}=-\mathrm{d} \dot{r}$ [131]. Obviously, in the Schwarzschild coordinate, the adiabatic invariant quantity of the spherically symmetric spacetime is derived by using the oscillating velocity of the black hole horizon. This is a key step to quantizing the entropy and the horizon area of the black hole. In the following subsection, we will continue the study of the adiabatic invariant quantity in the Painlevé coordinate.

\subsection{Adiabatic invariant in the Painlevé coordinate}

By introducing a shifting of the time coordinate, the coordinate singularity is removed at the black hole horizon in the Painlevé coordinate [132]. Here, when introducing the transformation $\mathrm{d} \tau^{\prime}=\mathrm{d} \tau+\frac{\sqrt{F(r)-1}}{F(r)} \mathrm{d} r$ into the metric (1), we have

$\mathrm{d} s^{2}=F(r) \mathrm{d} \tau^{\prime 2}+2 \sqrt{F(r)-1} \mathrm{~d} \tau^{\prime} \mathrm{d} r+\mathrm{d} r^{2}+r^{2} \mathrm{~d} \Omega_{n-2}^{2}$.

In the metric (14), there is not a coordinate singularity any more at the black hole horizon, and constant-time slices are just flat Euclidean spaces. These attractive features provide a superior setting for paths across the horizon. Similarly, when a particle tunnels out as described by this coordinate, the black hole horizon is shrinking by the velocity

$\dot{r_{h}}=-i(1-\sqrt{1-F(r)})$.

It is noteworthy that here the integral $\int_{q_{i}^{\text {in }}}^{q_{\text {out }}} p_{i}^{\text {out }} \mathrm{d} q_{i}$ has no contributions when doing the closed contour integral for the adiabatic invariant quantity $I=\oint p_{i} \mathrm{~d} q_{i}$. In a similar way, with the aid of the shrinking velocity of the black hole horizon (15), we have

$$
\begin{aligned}
I_{\text {adia }}^{p} & =\oint p_{i} \mathrm{~d} q_{i}=\int_{q_{i}^{\text {out }}}^{q_{i}^{\text {in }}} p_{i}^{\text {in }} \mathrm{d} q_{i}=2 \int_{r_{\text {out }}}^{r_{\text {in }}} \int_{0}^{H} \frac{\mathrm{d} H^{\prime}}{\dot{r}_{h}} \mathrm{~d} r_{h} \\
& =-2 i \int_{r_{\text {out }}}^{r_{\text {in }}} \int_{0}^{H} \frac{\mathrm{d} H^{\prime}}{1-\sqrt{1-F(r)}} \mathrm{d} r .
\end{aligned}
$$

In the Painlevé coordinate, we can see that the form of the adiabatic invariant quantity (16) is different from the one (13) that is obtained in the Schwarzschild coordinate. At first sight, it seems that the adiabatic invariant quantity $I_{\text {adia }}=$ $\oint p_{i} \mathrm{~d} q_{i}$ depends on the choice of coordinates. However, this is not the case. Further study shows that, when performing the integral (13) and (16) with respect to $r$, there is a pole at the horizon in (13) and (16). To avoid it, we have to get rid of it by taking a contour integral over a small half-loop going above the pole from right to left. In this case, by using the residue principle near the horizon, the integrals of Eqs. (13) and (16) can be written as

$$
\begin{aligned}
I_{\text {adia }}^{S} & =-4 i \int_{r_{\text {out }}}^{r_{\text {in }}} \int_{0}^{H} \frac{\mathrm{d} H^{\prime}}{F(r)} \mathrm{d} r=4 \pi \int_{0}^{H} \frac{\mathrm{d} H^{\prime}}{\left.\partial_{r} F(r)\right|_{r=r_{h}}}, \\
I_{\text {adia }}^{p} & =-2 i \int_{r_{\text {out }}}^{r_{\text {in }}} \int_{0}^{H} \frac{\mathrm{d} H^{\prime}}{1-\sqrt{1-F(r)}} \mathrm{d} r \\
& =4 \pi \int_{0}^{H} \frac{\mathrm{d} H^{\prime}}{\left.\partial_{r} F(r)\right|_{r=r_{h}}} .
\end{aligned}
$$

In this sense, we have

$$
\begin{aligned}
I_{\text {adia }}^{S} & =I_{\text {adia }}^{p}=4 \pi \int_{0}^{H} \frac{\mathrm{d} H^{\prime}}{\left.\partial_{r} F(r)\right|_{r=r_{h}}} \\
& =2 \pi \int_{0}^{H} \frac{\mathrm{d} H^{\prime}}{\kappa}=\hbar \int_{0}^{H} \frac{\mathrm{d} H^{\prime}}{T_{b h}}=\hbar S_{b h} .
\end{aligned}
$$

Note that we use the near-horizon approximation $F(r)=$ $\left.\partial_{r} F(r)\right|_{r=r_{h}}\left(r-r_{h}\right)$ in (17). Here, the surface gravity and the Hawking temperature of the black hole are given by $\kappa=$ $\frac{\left.\partial_{r} F(r)\right|_{r=r_{h}}}{2}$ and $T_{b h}=\hbar \kappa /(2 \pi)$, and in the last step the first law of the black hole thermodynamics $S_{b h}=\int_{0}^{H} \mathrm{~d} H^{\prime} / T$ has been employed to obtain the above findings, and here $S_{b h}$ is the black hole entropy. Obviously, from Eq. (18), one can see that, after doing the integral (13) and (16) with respect to $r$, the adiabatic invariant (8) in different types of coordinates indeed has the same formula, and finally this formula can be expressed as $I_{\text {adia }}=\hbar S_{b h}$. Hence, it is true that the adiabatic invariant quantity $\oint p_{i} \mathrm{~d} q_{i}$ is canonically invariant, which is fully in consistence with that of [1].

\section{Black hole spectroscopy in different gravity theories}

In the tunneling picture, with the aid of the oscillating velocity of the black hole horizon, we have obtained the unified form of adiabatic invariant quantity in different types (Schwarzschild and Painlevé) of coordinates as well as in different gravity frames. However, the black hole spectroscopy is not yet known. In the following, we will continue our study of the entropy/area spectrum for the black hole in different gravity theories.

\subsection{Black hole spectroscopy in Einstein gravity}

To obtain the black hole spectroscopy, we should first implement the Bohr-Sommerfield quantization rule to the adiabatic invariant quantity (18). In the semiclassical limit, it is

$I_{\text {adia }}=\oint p_{i} \mathrm{~d} q_{i}=n h=2 \pi n \hbar, \quad n \gg 1$. 
For a black hole in Einstein gravity (2), after substituting this rule (19) into Eq. (18), it is straightforward to show that the black hole entropy spectrum takes the form $S_{b h}=2 \pi n, n \gg$ 1. Obviously, the black hole entropy in Einstein gravity is evenly quantized and the spacing of the entropy spectrum is

$\Delta S_{b h}=2 \pi$

which is the same as the one given in $[133,134]$. Using the relation $S_{b h}=A /\left(4 \ell_{p}^{2}\right)$, the spacing of the area spectrum can be expressed as

$$
\Delta A=8 \pi \ell_{p}^{2}
$$

In Einstein gravity, our results show that the entropy and area of a global monopole black hole are quantized with an equally spaced spectrum fully in consistence with the one given by $[1,7]$ if one treats the action $I=\oint p_{i} \mathrm{~d} q_{i}$ as an adiabatic invariant quantity. In addition, the black hole parameter $\eta_{0}$ has no effects on the quantization of entropy and area.

\subsection{Black hole spectroscopy in EGB gravity}

Similarly, in EGB gravity, the spacing of the entropy spectrum in higher-dimensional spacetime reads

$\Delta S_{b h}=2 \pi$

According to Eq. (5), we can now derive the spacing of the horizon area

$$
\begin{aligned}
& \Delta A=A_{n+1}-A_{n}=8 \pi \ell_{p}^{2}+g\left(A_{n, n+1}\right), \\
& g\left(A_{n, n+1}\right)=6 \alpha \cdot \Sigma_{3}^{\frac{2}{3}} \cdot\left(A_{n}^{\frac{1}{3}}-A_{n+1}^{\frac{1}{3}}\right) .
\end{aligned}
$$

In EGB gravity, it is obvious that the entropy spectrum is also equally spaced with the form $\Delta S_{b h}=2 \pi$. So, one can see that the entropy spectrum is independent of gravity theories, and the dimension of spacetime has no effects on the quantization of the black hole entropy. This is compatible with the previous findings [50-52]. Since the trivial relationship $S_{b h}=A /\left(4 \ell_{p}^{2}\right)$ between horizon area and associated entropy is broken, the area spacing in EGB gravity is not equidistant as a result of the function $g\left(A_{n, n+1}\right)$. However, if we set the coupling constant $\alpha \rightarrow 0$, Eq. (5) will reduce to the linear relationship $S_{b h}=A /\left(4 \ell_{p}^{2}\right)$, and then the equidistant area spectrum will be recovered naturally [50-52].

\subsection{Black hole spectroscopy in HL gravity}

In a similar way, for a black hole in Hořava-Lifshitz gravity (6), the spacing of the entropy spectrum is

$\Delta S_{\mathrm{bh}}=2 \pi$
Recalling Eq. (7), we have

$\Delta A=A_{n+1}-A_{n}=8 \pi \ell_{p}^{2}+g\left(A_{n, n+1}\right)$,

$g\left(A_{n, n+1}\right)=\frac{4 \pi}{\omega} \ln \left[\frac{A_{n}}{A_{n+1}}\right]$.

Here, the logarithmic term that has been discussed in detail in $[128,129]$ is a small correction to the spacing of the area spectrum, which represents the feature of the HL gravity. Similarly to the EGB case, though the entropy spectrum in HL gravity is discrete and equally spaced, this does not hold true for the horizon area any longer. Our result is in agreement with that of $[128,129]$. Of course, by setting the limit $\omega \rightarrow$ $\infty$, the equidistant area spectrum also can be seen since the function $g\left(A_{n, n+1}\right) \sim 0$.

To conclude, a black hole in different gravity frames has the universal behavior of an equally spaced entropy spectrum: $\Delta S=2 \pi$. The entropy quantum is not only independent of the black hole parameter and the spacetime dimension, but also independent of different gravity theories. For the horizon area quantum, although the equally spaced spectrum can be found in Einstein gravity, this does not hold true in EGB gravity and HL gravity any longer.

\section{Action variable and adiabatic invariant}

It is well known that, for a physical system governed by a Hamiltonian $H$, if a dynamical quantity $I$ changes little during the time $t$ while $H$ accumulates a significant total change, the quantity $I$ is an adiabatic invariant. Ehrenfest showed that, for a quasiperiodic system, all Jacobi action integrals of the form $I=\oint p \mathrm{~d} q$ are action variables [9]. Moreover, Kwon and Nam pointed out that an action variable of the classical system is an adiabatic invariant, but an adiabatic invariant is not necessarily an action variable. The Bohr-Sommerfeld quantization rule can be applied only for the action variable of the classical system [26-28], rather than the adiabatic invariant. In this case, it was later showed by quasinormal modes that the area spectra of a rotating BTZ black hole is equally spaced [26-28].

In the tunneling framework, the oscillation of the black hole horizon can be assumed to be periodical [2]. In this sense, it is reasonable to apply the Bohr-Sommerfeld quantization rule to the action variable $I_{\text {adia }}=\oint p \mathrm{~d} q$. Finally, one has

$I_{\text {adia }} \propto \oint p \mathrm{~d} q \propto \int \frac{\mathrm{d} H}{T} \propto S_{b h}$.

It means that the black hole entropy is indeed an adiabatic invariant action variable (not only an adiabatic invariant, but also an action variable of the black hole.) [41,42]. But in different gravity theories, it is easy to see that the entropy 
is only a function of the horizon area of the black hole $A=$ $F\left(S_{b h}\right)$. In this sense, we confirm that the horizon area is only an adiabatic invariant rather than an action variable, thereby the quantum of the black hole entropy is more natural than that of the horizon area.

\section{Conclusion and discussion}

In this paper, we first discuss the adiabatic invariant quantity in different types of coordinates. In the tunneling picture, this quantity is obtained with the aid of the oscillating velocity of the black hole horizon. The result shows that the adiabatic invariant quantity in different types of coordinates has the same form, which can be finally expressed as $I_{\text {adia }}=\oint p \mathrm{~d} q=\hbar S_{b h}$. Then, using this adiabatic invariant, we further discuss the entropy and area spectrum of the black hole in different gravity frames. Our results show that the spacings of the entropy spectrum are all equidistant with the form of $\Delta S_{b h}=2 \pi$. It means that the entropy quantum is not only independent of the choice of coordinates, but also independent of different gravity theories. And, for the horizon area quantum, although the equally spaced spectrum can be found in Einstein gravity, this does not hold true in EGB gravity and HL gravity any longer. If we set the limit $\alpha \rightarrow 0, \omega \rightarrow \infty$, the original Bekenstein spacing of the area spectrum $\Delta A=8 \pi \ell_{p}^{2}$ could be recovered naturally. Finally, we interestingly find in more general gravity frames that the entropy of the black hole is an adiabatic invariant action variable, but the horizon area is only an adiabatic invariant. In this sense, we emphasize the concept that the quantum of the black hole entropy is more natural than that of the black hole area.

In our work, the observation of the black hole spectroscopy is mainly based on the semiclassical tunneling framework. However, the real physical progress should go beyond the semiclassical approximation, and the corresponding physical quantity would undergo a higher-order quantum corrections [43-45]. Hence, it is interesting for us to discuss quantum corrections of the black hole spectroscopy by improving Jiang-Han's proposal in future work.

Acknowledgements This work is supported by the Program for NCET12-1060, by the Sichuan Youth Science and Technology Foundation with Grant No. 2011JQ0019, by FANEDD with Grant No. 201319, by the Innovative Research Team in College of Sichuan Province with Grant No. 13TD0003, by the Sichuan Natural Science Foundation with Grant No. 16ZB0178, and by the starting fund of China West Normal University with Grant No. 14D014.

Open Access This article is distributed under the terms of the Creative Commons Attribution 4.0 International License (http://creativecomm ons.org/licenses/by/4.0/), which permits unrestricted use, distribution, and reproduction in any medium, provided you give appropriate credit to the original author(s) and the source, provide a link to the Creative Commons license, and indicate if changes were made. Funded by SCOAP ${ }^{3}$.

\section{References}

1. Q.Q. Jiang, Y. Han, Phys. Lett. B 718, 584 (2012)

2. B.R. Majhi, E.C. Vagenas, Phys. Lett. B 701, 623 (2011)

3. J.D. Bekenstein, Lett. Nuovo Cim. 4, 737 (1972)

4. V.F. Mukhanov, JETP Lett. 44, 63 (1986)

5. J. Louko, J. Makela, Phys. Rev. D 54, 4982 (1996)

6. S. Hod, Phys. Rev. D 59, 024014 (1999)

7. J.D. Bekenstein, Phys. Rev. D 7, 2333 (1973)

8. J.D. Bekenstein, Lett. Nuovo Cim. 11, 476 (1974)

9. J.D. Bekenstein (1997). arXiv:gr-qc/9710076

10. J.D. Bekenstein (1998). arXiv:gr-qc/9808028

11. J.D. Bekenstein (1998). arXiv: gr-qc/9805045

12. G. Kunstatter, Phys. Rev. Lett. 90, 161301 (2003)

13. S. Hod, Phys. Rev. Lett. 81, 4293 (1998)

14. S. Hod, Gen. Relat. Gravit. 31, 1639 (1999)

15. J.D. Bekenstein, V.F. Mukhanov, Phys. Lett. B 360, 7 (1995)

16. A. Barvinsky, S. Das, G. Kunstatter, Class. Quant. Gravit. 18, $4845(2001)$

17. A. Barvinsky, S. Das, G. Kunstatter, Phys. Lett. B 517, 415 (2001)

18. A. Barvinsky, S. Das, G. Kunstatter, Found. Phys. 32, 1851 (2002)

19. M.R. Setare, Phys. Rev. D 69, 044016 (2004)

20. M.R. Setare, Class. Quant. Gravit. 21, 1453 (2004)

21. M.R. Setare, Gen. Relat. Gravit. 37, 1411 (2005)

22. M.R. Setare, E.C. Vagenas, Mod. Phys. Lett. A 20, 1923 (2005)

23. M. Maggiore, Phys. Rev. Lett. 100, 141301 (2008)

24. E.C. Vagenas, J. High Energy Phys. 0811, 073 (2008)

25. Y.S. Myung, Phys. Lett. B 689, 42 (2010)

26. Y. Kwon, S. Nam, Class. Quant. Gravit. 27, 125007 (2010)

27. Y. Kwon, S. Nam, Class. Quant. Gravit. 27, 165011 (2010)

28. Y. Kwon, S. Nam, Class. Quant. Gravit. 28, 035007 (2011)

29. A.J.M. Medved, Class. Quant. Gravit. 25, 205014 (2008)

30. D. Kothawala, T. Padmanabhan, S. Sarkar, Phys. Rev. D 78, 104018 (2008)

31. S.W. Wei, Y.X. Liu, K. Yang, Y. Zhong, Phys. Rev. D 81, 104042 (2010)

32. S. Fernando, Phys. Rev. D 79, 124026 (2009)

33. R.G. Daghigh, M.D. Green, Class. Quant. Gravit. 26, 125017 (2009)

34. A. Lopez-Ortega, Class. Quant. Gravit. 28, 035009 (2011)

35. K. Ropotenko, Phys. Rev. D 82, 044037 (2010)

36. D.Y. Chen, H.T. Yang, X.T. Zu, Eur. Phys. J. C 69, 289 (2010)

37. H.L. Li, R. Lin, Eur. Phys. J. C 73(2), 2316 (2013)

38. J. Suresh, V.C. Kuriakose, Eur. Phys. J. C 75, 214 (2015)

39. K.S. Gupta, E. Harikumar, T. Jurić, S. Meljanac, A. Samsarov, J. High Energy Phys. 1509, 025 (2015)

40. I. Sakalli, Eur. Phys. J. C 75, 144 (2015)

41. X.X. Zeng, X.M. Liu, W.B. Liu, Eur. Phys. J. C 72, 1967 (2012)

42. X.X. Zeng, W.B. Liu, Eur. Phys. J. C 72, 1987 (2012)

43. Q.Q. Jiang, Y. Han, X. Cai, J. High Energy Phys. 1008, 049 (2010)

44. Q.Q. Jiang, X. Cai, J. High Energy Phys. 1011, 066 (2010)

45. Q.Q. Jiang, Eur. Phys. J. C 72, 2086 (2012)

46. R. Banerjee, B.R. Majhi, E.C. Vagenas, Europhys. Lett. 92, 20001 (2010)

47. K. Parattu, B.R. Majhi, T. Padmanabhan, Phys. Rev. D 87, 124011 (2013)

48. A. Bakshi, B.R. Majhi, S. Samanta, Phys. Lett. B 765, 334 (2017)

49. B.R. Majhi, Phys. Lett. B 686, 49 (2010)

50. D. Kothawala, T. Padmanabhan, S. Sarkar, Phys. Rev. D 78, $104018(2008)$

51. S.W. Wei, R. Li, Y.X. Liu, J.R. Ren, J. High Energy Phys. 0903, 076 (2009)

52. R. Banerjee, B.R. Majhi, E.C. Vagenas, Phys. Lett. B 686, 279 (2010)

53. Q.Q. Jiang, S.Q. Wu, Phys. Lett. B 635, 151 (2006) 
54. Q.Q. Jiang, S.Q. Wu, X. Cai, Phys. Rev. D 73, 064003 (2006)

55. S.Q. Wu, Q.Q. Jiang, J. High Energy Phys. 0603, 079 (2006)

56. S.W. Zhou, W.B. Liu, Phys. Rev. D 77, 104021 (2008)

57. Q.Q. Jiang, X. Cai, J. High Energy Phys. 0911, 110 (2009)

58. J. Hu, H. Yu, J. High Energy Phys. 1209, 062 (2012)

59. Y.P. Hu, J.J. Zhang, Z. Zhao, Mod. Phys. Lett. A 21, 2143 (2006)

60. W.B. Liu, Phys. Lett. B 634, 541 (2006)

61. Z.Z. Ma, Phys. Lett. B 666, 376 (2008)

62. Z.Z. Ma, Class. Quant. Gravit. 26, 045002 (2009)

63. P. Xu, Z. Wang, Y. Han, Class. Quant. Gravit. 28, 125012 (2011)

64. H. Kim, Phys. Lett. B 703, 94 (2011)

65. L. Zhao, Commun. Theor. Phys. 47, 835 (2007)

66. D. Gangopadhyay, G. Manna, Europhys. Lett. 100, 49001 (2012)

67. M. Sharif, W. Javed, Eur. Phys. J. C 72, 1997 (2012)

68. M. Angheben, M. Nadalini, L. Vanzo, S. Zerbini, J. High Energy Phys. 05, 014 (2005)

69. M. Nadalini, L. Vanzo, S. Zerbini, J. Phys. A 39, 6601 (2006)

70. R.D. Criscienzo, M. Nadalini, L. Vanzo, S. Zerbini, G. Zoccatelli, Phys. Lett. B 657, 107 (2007)

71. S.H. Mehdipour, Phys. Rev. D 81, 124049 (2010)

72. M.A. Rahman, M.I. Hossain, Phys. Lett. B 712, 1 (2012)

73. R.D. Criscienzo, L. Vanzo, Europhys. Lett. 82, 60001 (2008)

74. R. Li, J.R. Ren, Phys. Lett. B 661, 370 (2008)

75. R. Li, J.R. Ren, Class. Quant. Gravit. 25, 125016 (2008)

76. R. Kerner, R.B. Mann, Class. Quant. Gravit. 25, 095014 (2008)

77. R. Kerner, R.B. Mann, Phys. Lett. B 665, 277 (2008)

78. R.D. Criscienzo, S.A. Hayward, M. Nadalini, L. Vanzo, S. Zerbini (2009). arXiv:0906.1725

79. S. Bhattacharya, A. Saha, Gen. Relat. Gravit. 42, 1809 (2010)

80. J. Ahmed, K. Saifullah, J. Cosmol. Astropart. Phys. 11, 023 (2011)

81. J. Ahmed, K. Saifullah, J. Cosmol. Astropart. Phys. 08, 011 (2011)

82. D.Y. Chen, Q.Q. Jiang, X.T. Zu, Phys. Lett. B 665, 106 (2008)

83. Q.Q. Jiang, Phys. Rev. D 78, 044009 (2008)

84. Q.Q. Jiang, Phys. Lett. B 666, 517 (2008)

85. D.Y. Chen, Q.Q. Jiang, S.Z. Yang, X.T. Zu, Class. Quant. Gravit. 25, 205022 (2008)

86. H.L. Li, W.Y. Qi, R. Lin, Phys. Lett. B 677, 332 (2009)

87. D.Y. Chen, H. Yang, X.T. Zu, Phys. Lett. B 681, 463 (2009)

88. K. Lin, S.Z. Yang, Phys. Rev. D 79, 064035 (2009)

89. K. Lin, S.Z. Yang, Phys. Lett. B 674, 127 (2009)

90. R. Banerjee, B.R. Majhi, J. High Energy Phys. 06, 095 (2008)

91. S.K. Modak, Phys. Lett. B 671, 167 (2009)

92. B.R. Majhi, Phys. Rev. D 79, 044005 (2009)

93. R. Banerjee, B.R. Majhi, D. Roy, arXiv:0901.0466

94. R. Banerjee, S.K. Modak, J. High Energy Phys. 05, 063 (2009)

95. J.Y. Zhang, Phys. Lett. B 668, 353 (2008)

96. R.G. Cai, L.M. Cao, Y.P. Hu, J. High Energy Phys. 08, 090 (2008)

97. T. Zhu, J.R. Ren, Eur. Phys. J. C 62, 413 (2009)

98. T. Zhu, J.R. Ren, M.F. Li, J. Cosmol. Astropart. Phys. 08, 010 (2009)
99. R. Banerjee, S.K. Modak, J. High Energy Phys. 11, 073 (2009)

100. R. Banerjee, B.R. Majhi, Phys. Lett. B 675, 243 (2009)

101. R. Banerjee, B.R. Majhi, Phys. Lett. B 674, 218 (2009)

102. B. Zhang, Q.Y. Cai, L. You, M.S. Zhan, Phys. Lett. B 675, 98 (2009)

103. Y.X. Chen, K.N. Shao, Phys. Lett. B 678, 131 (2009)

104. R.D. Criscienzo, L. Vanzo, S. Zerbini, J. High Energy Phys. 05, $092(2010)$

105. D. Singleton, E.C. Vagenas, T. Zhu, J.R. Ren, J. High Energy Phys. 08, 089 (2010). [Erratum ibid. 1101(2011) 021]

106. S.P. Kim, J. High Energy Phys. 09, 054 (2010)

107. S.D. Mathur, Class. Quant. Gravit. 28, 125010 (2011)

108. K. Nozari, S. Saghafi, J. High Energy Phys. 11, 005 (2012)

109. A. Zampeli, D. Singleton, E.C. Vagenas, J. High Energy Phys. 06, 097 (2012)

110. M. Stone, Class. Quant. Gravit. 30, 085003 (2013)

111. B. Zhang, Q.Y. Cai, M.S. Zhan, L. You, Phys. Rev. D 87, 044006 (2013)

112. Q.Q. Jiang, S.Z. Yang, S.Q. Wu, Int. J. Theor. Phys. 45, 2274 (2006)

113. Q.Q. Jiang, S.Z. Yang, H.L. Li, Int. J. Theor. Phys. 45, 1757 (2006)

114. Q.Q. Jiang, W. Ren, J. Tang, X.F. Liu, Int. J. Theor. Phys. 46, 1449 (2007)

115. S.Z. Yang, Q.Q. Jiang, H.L. Li, Int. J. Theor. Phys. 46, 625 (2007)

116. S.Z. Yang, Q.Q. Jiang, Int. J. Theor. Phys. 46, 2138 (2007)

117. Q.Q. Jiang, Y. Han, X. Cai, J. High Energy Phys. 08, 049 (2010). [Erratum ibid. 06 (2012) 120]

118. Q.Q. Jiang, X. Cai, J. High Energy Phys. 11, 066 (2010). [Erratum ibid. 06 (2012) 118]

119. J. Pu, Y. Han, Int. J. Theor. Phys. 55, 5077 (2016)

120. J. Pu, Y. Han, Int. J. Theor. Phys. 56, (in press) (2017)

121. M. Barriola, A. Vilenkin, Phys. Rev. Lett. 63, 341 (1989)

122. H. Yu, Phys. Rev. D 65, 087502 (2002)

123. D.G. Boulware, S. Deser, Phys. Rev. Lett. 55, 2656 (1985)

124. P. Hořava, Phys. Rev. D 79, 084008 (2009)

125. P. Hořava, J. High Energy Phys. 0903, 020 (2009)

126. J.J. Peng, S.Q. Wu, Eur. Phys. J. C 66, 325 (2010)

127. A. Kehagias, K. Sfetsos, Phys. Lett. B 678, 123 (2009)

128. Y.S. Myung, Phys. Lett. B 678, 127 (2009)

129. Y.S. Myung, Phys. Lett. B 684, 158 (2010)

130. M.K. Parikh, F. Wilczek, Phys. Rev. Lett. 85, 5042 (2000)

131. J.Y. Zhang, Z. Zhao, Phys. Rev. D 83, 064028 (2011)

132. P. Painlevé, Compt. Rend. Acad. Sci. (Paris) 173, 677 (1921)

133. H.A. Kastrup, Phys. Lett. B 385, 75 (1996)

134. A. Barvinsky, G. Kunstatter, Phys. Lett. B 389, 231 (1996). arXiv:gr-qc/9607030 